\title{
Análise do Sentimento de Mensagens de Chats em uma Turma de Graduação de um Curso de Educação à Distância
}

\author{
Emanuel Ferreira Coutinho ${ }^{1}$, Leonardo O. Moreira $^{1}$ \\ Gabriel A. L. Paillard ${ }^{1}$, Ernesto T. Lima Neto ${ }^{1}$ \\ ${ }^{1}$ Instituto Universidade Virtual (UFC Virtual) \\ Universidade Federal do Ceará (UFC) - Fortaleza - CE - Brasil \\ \{emanuel, leoomoreira, gabriel, ernesto\}evirtual.ufc.br
}

\begin{abstract}
The task of analyzing people emotions and feelings in relation to entities is known as sentiment analysis. This activity search to extract feelings present in subjective texts, analyze in a computational way and understand if such information is positive, negative or neutral. The Naive Bayes is a simple probabilistic classifier based on the application of Bayes' theorem, commonly used in text classification. This work aims to analyze the sentiment of text messages. A case study was designed to analyze the sentiment of chat messages captured from a Virtual Learning Environment.
\end{abstract}

Resumo. A tarefa de analisar emoções e sentimentos das pessoas em relação a entidades é conhecida como Análise de Sentimento. Esta atividade busca extrair sentimentos presentes em textos subjetivos, analisar de maneira computacional e entender se tal informação é positiva, negativa ou neutra. O Naive Bayes é um simples classificador probabilístico baseado na aplicação do teorema de Bayes, comumente utilizado na classificação de textos. Este trabalho tem como objetivo analisar o sentimento de mensagens de texto. Um estudo de caso foi projetado para analisar o sentimento das mensagens de um chat capturadas de um Ambiente Virtual de Aprendizagem.

\section{Introdução e Motivação}

Dentro da área de Recuperação da Informação, a tarefa de analisar 'opiniões, sentimentos, avaliações, apreciações, atitudes e emoções das pessoas em relação a entidades como produtos, serviços, organizações, indivíduos, problemas, eventos, tópicos e seus atributos" é conhecida por Análise de Sentimento [Liu 2012]. Esta atividade procura extrair sentimentos presentes em textos, que normalmente são subjetivos, e computacionalmente analisar e entender se tal informação transmite uma opinião positiva, negativa ou neutra sobre um determinado assunto ou objeto de interesse [Missen et al. 2013].

Desse modo, o trabalho que auxilia a determinar automaticamente a direção do sentimento (positivo ou negativo) em textos é frequentemente designado de Análise de Sentimento ou Opinion Mining [Li and Liu 2010].

O Naive Bayes é um simples classificador probabilístico baseado na aplicação do teorema de Bayes. Ele é frequentemente utilizado como base na classificação de textos por ser rápido e fácil de se implementar [Rennie et al. 2003]. Gomes et al. (2013) mencionam que o classificador Naive Bayes é considerado um dos mais eficientes em 
V Congresso Brasileiro de Informática na Educação (CBIE 2016)

Anais dos Workshops do V Congresso Brasileiro de Informática na Educação (CBIE 2016)

aspectos relacionados ao processamento e precisão na classificação de novas amostras [Gomes et al. 2013].

Diversos estudos no contexto de redes sociais estão focados na identificação e monitoramento de polaridade em mensagens compartilhadas, partindo da hipótese que a quantidade expressiva de dados postados uma parcela significante estaria relacionada ao humor e a emoções expressas pelos usuários [Araújo et al. 2013].

Este trabalho tem como objetivo analisar o sentimento de mensagens de texto. Um estudo de caso foi projetado e executado com mensagens de um chat capturadas de um Ambiente Virtual de Aprendizagem (AVA) para a análise do sentimento.

O restante do trabalho está dividido nas seguintes seções: na Seção 2 alguns trabalhos relacionados são apresentados; a Seção 3 descreve a metodologia adotada neste trabalho; na Seção 4 os resultados do experimento e sua análise são descritos; e por fim, na Seção 5 as conclusões e trabalhos futuros são apresentados.

\section{Trabalhos Relacionados}

Diversos trabalhos na literatura discutem a análise de sentimento em diversos tipos de textos, tais como fóruns e chats.

Wordnet consiste basicamente em uma base de dados láxica que fornece a seus usuários um conjunto de substantivos, verbos, adjetivos e advérbios em inglês, organizados em grupos de sinônimos [Oliveira and Roman 2013]. Cada uma das diversas formas de interações, como exemplo recomendação de notícias, reconhecimento automático de inferência em textos e classificação de documentos, exige uma API (Interface de Programação de Aplicação - Application Programming Interface) especializada, para a conexão da aplicação com o Wordnet. Por tratar-se de um mapeamento com outra língua, possivelmente essa abordagem pode perder alguma informação durante a tradução, sendo o ideal a existência de uma API para cada tipo de acesso ao Wordnet. Oliveira e Roman (2013) apresentarem neste trabalho a JWN-Br, uma API para a Wordnet.br, desenvolvida em Java. Suas vantagens são: (i) fornecer acesso direto a Wordnet.br estruturando seus dados sob a forma de objetos Java; (ii) abstrair a codificação interna usada pela Wordnet.br, tornando seu uso mais fácil ao programador; e (iii) possibilitar o incremento local, por meio da API, da base de dados original da Wordnet.br.

Diversos métodos foram propostos na literatura não para analisar mensagens curtas em tempo real, mas para analisar sentenças longas. POrém, poucos esforços foram realizados na comparação de tais métodos [Araújo et al. 2013]. O objetivo deste trabalho foi comparar métodos para análise de sentimento, utilizando duas diferentes bases de dados provenientes de redes sociais para comparar oito métodos propostos na literatura: LIWC, Happiness Index, SentiWordNet, SASA, PANAS-t, Emoticons, SenticNet e SentiStrength. Como resultados deste gtrabalho, obteve-se que os métodos possuem diferentes graus de abrangência, que nenhum método alcançou níveis altos de abrangência e concordância ao mesmo tempo, e existe desacordo entre os métodos na predição de sentimentos para diferentes eventos.

A identificação automática de emoções em textos tem apresentado resultados significativos em diversas aplicações [Dosciatti et al. 2013]. Dosciatti et al. (2013) apresentaram uma abordagem utilizando Máquinas de Vetores de Suporte (SVM) na identificação 
de emoções em textos escritos em Português do Brasil. A base de dados utilizada no experimento foi composta por notícias extraídas de um jornal online. Os textos foram previamente rotulados e submetidos a um classificador SVM com configuração multiclasse, obtendo uma taxa de acerto de $61 \%$.

As redes sociais se tornaram um meio global de comunicação com uma quantidade muito grande de usuários [Malheiros and Lima 2013]. Esta riqueza de informaçes públicas tem atraído a atenção de instituições e empresas que desejam conhecer melhor o que as pessoas estão pensando sobre elas. Malheiros e Lima (2013) apresentaram uma ferramenta de análise de sentimentos para mensagens compartilhadas em redes sociais utilizando o SenticNet como base de conhecimento. Os primeiros experimentos realizados para avaliação da ferramenta mostraram que a abordagem consegue classificar mensagens adequadamente e suporta a grande quantidade e velocidade na qual elas são enviadas para as redes sociais.

O estudo e compreensão de como a afetividade influencia no desenvolvimento da cognição humana tem forte impacto em contextos educacionais à distância [David et al. 2014]. David et al. (2014) apresentaram uma pesquisa para o desenvolvimento um modelo computacional baseado em ontologias capaz de identificar a manifestação de interações visando o aprendizado em fóruns de discussão. A análise ocorreu com base no Sistema de Análise de Interações Contingentes (SAIC), composto por categorias que avaliam diferentes facetas da comunicação online. Os primeiros passos para a produção de uma ontologia de domínio foram descritos, com a categoria Afetividade para a criação de um sistema capaz de observar se as mensagens trocadas entre os estudantes de cursos à distância concorrem de fato para o aprendizado individual e coletivo.

A análise de sentimentos tornou-se uma ferramenta essencial para aplicação em diversos contextos, incluindo análise de opinião do usuário sobre produtos e serviços, previsão em campanhas políticas e tendências do mercado financeiro [Reis et al. 2015]. Apesar do grande interesse neste tema e na quantidade de pesquisas na área, a maioria dos métodos foram projetados para funcionar com palavras em inglês. Reis et al. (2015) propuseram uma abordagem para a utilização de determinados métodos para análise de sentimentos em nove diferentes linguas. Para cada idioma foram utilizadas bases de dados previamente rotuladas e uma simples tradução automática para o inglês. Desenvolveu-se uma metodologia para comparar e validar os resultados, e os resultados demonstram o potencial desta abordagem para tornar a análise de sentimentos independente da língua inglesa.

David et al. (2015) apresentaram um modelo computacional baseado em ontologias para a representação do diálogo freireano e sua posterior implementação em AVA, tendo em vista a análise de processos interacionais da EaD [David et al. 2015]. Buscando-se favorecer a realização de avaliações formativas na $\mathrm{EaD}$, esta pesquisa apresentou o desenvolvimento da ontologia do diálogo freireano, resultado de uma ampla investigação linguística que detectou seus principais marcadores em um fórum de discussão. A classificação das mensagens em categorias foi realizada a partir da análise de seu conteúdo, baseada na identificação de marcadores linguísticos. Por exemplo, se os termos "parabéns" (marca de elogio) e "obrigado" (marca de gratidão) forem encontrados na mensagem, pode-se dizer que existe a presença do parâmetro Afetividade. Por 
V Congresso Brasileiro de Informática na Educação (CBIE 2016)

Anais dos Workshops do V Congresso Brasileiro de Informática na Educação (CBIE 2016)

exemplo, uma mensagem pode possuir Afetividade Total, outra, Afetividade Parcial e uma terceira, Ausência de Afetividade.

Para sumarizar a compararção do trabalho proposto com os trabalhos relacionados, construiu-se a Tabela 1. O trabalho proposto neste artigo apenas utiliza uma técnica (Naive Bayes), e não aprofunda muito a classificação ou desempenho, pois seu objetivo é avaliar se a técnica é útil para a análise e principalmente para a viabilidade técnica. Os critérios de comparação são: se foram realizados ou não experimentos, se houve comparação entre diferentes médodos de análise, se o trabalho propôs algum framework, métodos ou APIs, se utilizou ontologias e por fim, qual foi o principal método ou técnica utilizado na pesquisa.

Tabela 1. Comparação com trabalhos relacionados. Critérios: Experimentos (Exper.), Comparação (Comp.), Frameworks, métodos e APIs (F/M/API), ontologias e método ou técnica Utilizado (M/T Utilizado)

\begin{tabular}{l|c|c|c|c|l}
\hline \hline Trabalho & Exper. & Comp. & F/M/API & Ontologias & M/T Utilizado \\
\hline [Oliveira and Roman 2013] & não & não & $\operatorname{sim}$ & não & WordNet e JWN-Br \\
\hline [Araújo et al. 2013] & $\operatorname{sim}$ & $\operatorname{sim}$ & $\operatorname{sim}$ & não & 8 diferentes métodos \\
\hline [Dosciatti et al. 2013] & $\operatorname{sim}$ & não & não & não & $\begin{array}{l}\text { Máquina de Vetores } \\
\text { de Suporte (SVM) }\end{array}$ \\
\hline [Malheiros and Lima 2013] & $\operatorname{sim}$ & $\operatorname{sim}$ & $\operatorname{sim}$ & não & SenticNet \\
\hline [David et al. 2014] & não & não & não & sim & Protégé \\
\hline [Reis et al. 2015] & $\operatorname{sim}$ & $\operatorname{sim}$ & não & sim & 8 diferentes métodos \\
\hline [David et al. 2015] & não & não & não & sim & Protégé \\
\hline Artigo proposto & $\operatorname{sim}$ & não & não & não & Naive Bayes \\
\hline
\end{tabular}

\section{Contextualização e Metodologia}

As subseções a seguir descrevem o contexto dos dados utilizados neste trabalho e a metodologia aplicada no experimento.

\subsection{Contextualização do Curso, Disciplina e Turma}

A Universidade Aberta do Brasil (UAB) ${ }^{1}$ é um sistema integrado por universidades públicas que oferece cursos de nível superior para a população com dificuldade de acesso à formação universitária, por meio da Educação à Distância (EAD). O público em geral é atendido, mas os professores que atuam na educação básica têm prioridade de formação, seguidos dos dirigentes, gestores e trabalhadores em educação básica. Atualmente, a UAB possui no estado do Ceará diversos cursos de graduação, com centenas de alunos matriculados em variadas cidades. Possui toda uma infraestrutura local e distribuída, espaços físicos cedidos por prefeituras, colégios e instituições federais, uma rede de comunicação para um AVA, uma equipe para apoio finaceiro e logístico, além de uma equipe de coordenadores de curso e disciplinas, tutores presenciais e à distância.

Dentre seus cursos de graduação, existe a Licenciatura em Letras Inglês, com duração de 4 anos e diploma expedido e registrado pela Universidade Federal do Ceará igual aos cursos presenciais. A disciplina se chama Educação a Distância e possui atividades de aulas virtuais, no AVA SOLAR ${ }^{2}$, de leitura e exercício, fóruns, chats e avaliações, e com carga-horária de 64 horas/aula.

\footnotetext{
${ }^{1}$ Universidade Aberta do Brasil (UAB) - http://uab.capes.gov.br/

${ }^{2}$ Sistema Online de Aprendizagem - http://www.solar.virtual.ufc.br/
} 
A avaliação da disciplina possuiu uma divisão quanto à atividades presenciais e virtuais, sendo atividades virtuais valendo $40 \%$ da média e atividades presenciais valendo $60 \%$ da média. Dentre as avaliações em atividades virtuais estão os trabalhos escritos enviados ao portfólio do aluno, fóruns e chats. O período da disciplina foi de janeiro a março de 2011, sendo apenas um pequeno estudo de caso com dados reais para validar a ideia de se analisar o sentimento dos chats.

Já que a disciplina possuia avaliação baseada nos textos de chats e fóruns, surgiu a ideia de se analisar como estava o clima de participação dos alunos e tentar identificar a necessidade de intervenção do professor/tutor para tentar ajustar o nível das discussões.

O chat 1 foi planejado para ser um primeiro encontro virtual para a apresentação do professor/tutor e alunos. Neste chat, o objetivo inicial era que os alunos tivessem um primeiro contato com o ambiente e conhecessem os demais colegas. $\mathrm{O}$ chat 2 e o chat 3 tiveram como objetivo discutir as aulas do período. Eram atividades onde o aluno deveria focar no conteúdo ministrado nas aulas presenciais e no conteúdo das aulas disponibilizadas no AVA. Por fim, o chat 4 foi praticamente uma conversa de revisão do conteúdo e discussão geral como seriam as avaliações presenciais finais.

\subsection{Metodologia}

A disciplina tem em seu planejamento chats que os alunos devem participar. Especificamente para esta turma ocorreram quatro chats: um de apresentação da turma, dois para discussão do conteúdo, e um para dúvidas gerais. A turma analisada possuiu 30 alunos.

O algoritmo de classificação utilizado neste experimento foi o Naive Bayes. Sua implementação foi realizada em Java.

$\mathrm{Na}$ análise do sentimento, uma tarefa é determinar a polaridade do texto: positiva, negativa ou neutra. $\mathrm{O}$ algoritmo Naive Bayes utiliza probabilidades para decidir qual categoria melhor se enquadra a um determinado texto de entrada. A decisão de classificação é baseada em um modelo obtido após o processo de treinamento. O treinamento do modelo é realizado por meio da análise da relação entre as palavras do texto de treinamento e suas categorias de classificação. O algoritmo é considerado "ingênuo" porque se supõe que o valor de uma característica particular é independente do valor de qualquer outra característica, dada a variável de classe.

Para o experimento utilizou-se o Apache Mahout ${ }^{3}$. O objetivo do projeto Apache Mahout é possibilitar a construção de um ambiente para rapidamente criar aplicações de aprendizagem de máquina escaláveis de elevada performance. O Mahout é uma biblioteca de aprendizagem de máquina de código aberto do Apache [Owen et al. 2011]. Ele possui uma implementação do algoritmo bayesiano utilizado na etapa de mineração de textos.

A base de treinamento utilizou 113 palavras, onde cada uma delas foi categorizada em positiva (1) e negativa (0). As mensagens de cada chat seriam categorizadas em positiva ou negativa manualmente e todas passariam pelo algoritmo que automaticamente classificaria cada mensagem. Com isso, é possível a comparação entre o considerado real do sentimento da mensagem e o obtido pela aplicação. Para ampliar um pouco o experimento, e validar a complexidade de analisar mais de um nível de sentimento, um

\footnotetext{
${ }^{3}$ Apache Mahout: Scalable machine learning and data mining - http://mahout.apache.org/
} 
V Congresso Brasileiro de Informática na Educação (CBIE 2016)

Anais dos Workshops do V Congresso Brasileiro de Informática na Educação (CBIE 2016)

pequeno experimento foi projetado apenas para o chat 1, com a adição da categoria "neutro", com a respectiva atualização do dicionário com esta categoria. Ao final, análises foram realizadas em cima dos dados, gráficos e tabelas.

\section{Resultados e Análise}

As subseções a seguir apresentam os resultados obtidos após o cálculo do sentimento das mensagens dos quatro chats, dispondo-os em uma tabela e gráficos de barras. Além disso, alguns comentários foram realizados para a análise dos dados.

\subsection{Resultados}

Em cada chat foi calculado para cada mensagem a probabilidade de ser uma mensagem negativa ou positiva. Realizou-se também um procedimento manual de análise de cada mensagem, onde se indicava se o teor da mensagem era neagtiva ou positiva. Esse procedimento possibilitou o cálculo da proporção de acertos do cálculo com uma análise manual.

A Tabela 2 exibe a quantidade de mensagens de cada chat, com seu respectivo parecer (positivo ou negativo), discriminados pelo resultado obtido pela aplicação, pela

Tabela 2. Quantidade de mensagens classificadas pela ferramenta e manualmente, quantidade de mensagens acertadas e parecer, para todos os chats

\begin{tabular}{|c|c|c|c|}
\hline \multicolumn{4}{|c|}{ CHAT 1} \\
\hline Parecer & Resultado do Classificador & Resultado Real & Quantidade de Acertos \\
\hline Negativo & 95 & 69 & 62 \\
\hline Positivo & 23 & 49 & 16 \\
\hline Total & 118 & 118 & 78 \\
\hline \multicolumn{4}{|c|}{ CHAT 2} \\
\hline Parecer & Resultado do Classificador & Resultado Real & Quantidade de Acertos \\
\hline Negativo & 556 & 464 & 423 \\
\hline Positivo & 101 & 193 & 60 \\
\hline Total & 657 & 657 & 483 \\
\hline \multicolumn{4}{|c|}{ CHAT 3} \\
\hline Parecer & Resultado do Classificador & Resultado Real & Quantidade de Acertos \\
\hline Negativo & 255 & 214 & 191 \\
\hline Positivo & 40 & 81 & 17 \\
\hline Total & 295 & 295 & 208 \\
\hline \multicolumn{4}{|c|}{ CHAT 4} \\
\hline Parecer & Resultado do Classificador & Resultado Real & Quantidade de Acertos \\
\hline Negativo & 308 & 257 & 241 \\
\hline Positivo & 62 & 113 & 46 \\
\hline Total & 370 & 370 & 287 \\
\hline \multicolumn{4}{|c|}{ CHAT 1 - Recalculado com três categorias } \\
\hline Parecer & Resultado do Classificador & Resultado Real & Quantidade de Acertos \\
\hline Negativo & 63 & 8 & 7 \\
\hline Positivo & 32 & 37 & 24 \\
\hline Neutro & 23 & 73 & 22 \\
\hline Total & 118 & 118 & 53 \\
\hline
\end{tabular}


V Congresso Brasileiro de Informática na Educação (CBIE 2016)

Anais dos Workshops do V Congresso Brasileiro de Informática na Educação (CBIE 2016)

classificação manual e a quantidade de acertos (quantas mensagens foram classificadas conforme a classificação manual). A linha de total de cada chat possui os mesmos valores para as colunas "Resultado do Classificador" e "Resultado Real", pois estas quantidades de mensagens são as mesmas, diferenciando-se apenas por serem as mensagens calculadas (categorias identificadas pela aplicação) e as mensagens reais (categorias identificadas manualmente), respectivamente.

A Figura 1 exibe gráficos que representam as mensagens dos quatro chats, suas quantidades de acertos e erros no cálculo, e suas taxas de acerto. A Figura 1(a) apresenta a quantidade de mensagens calculadas acertadamente. A soma desses dois valores corresponde apenas à quantidade de acertos positivos e negativos. A quantidade total de mensagens é maior, e essa diferença corresponde ao erro no cálculo do sentimento. A Figura 1(b) e (c) exibem a taxa de acertos por chat em valors percentuais, apenas se diferenciando por mostrar em relação apenas às mensagens com sentimento igual ao real (corretas) e ao total de mensagens respectivamente. Após o cálculo do sentimento dos quatro chats, executou-se apenas para o chat 1 um novo cálculo, adicionando o tipo "neutro" às possibilidades (negativo e positivo). A Figura 1(d) exibe a quantidade de mensagens discriminadas pelo resultado do cálculo, a quantidade real obtida pelo processo manual e a quantidade de acertos para o chat 1 recalculado.

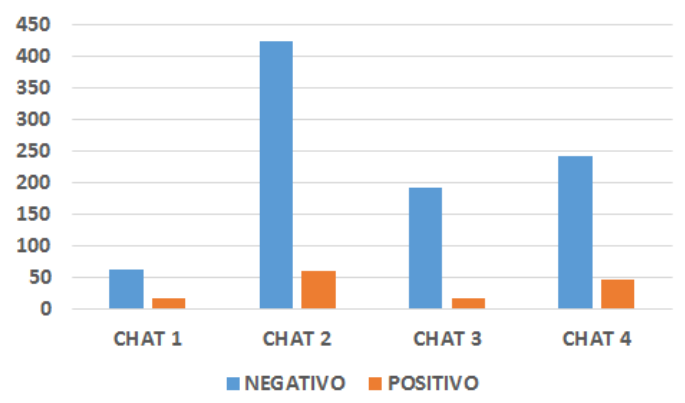

(a) Quantidade de acertos obtidos

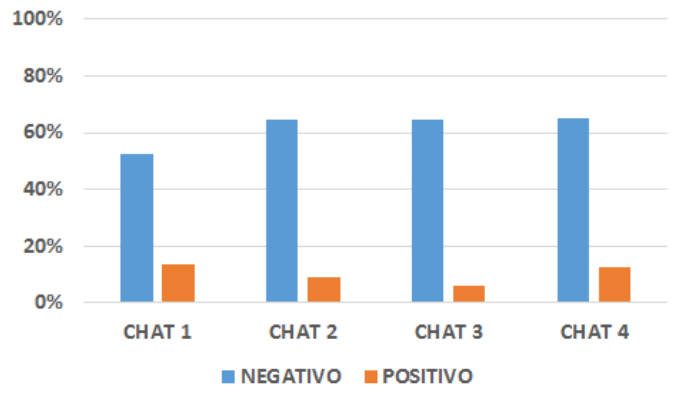

(c) Percentual de acertos sobre o total de mensagens

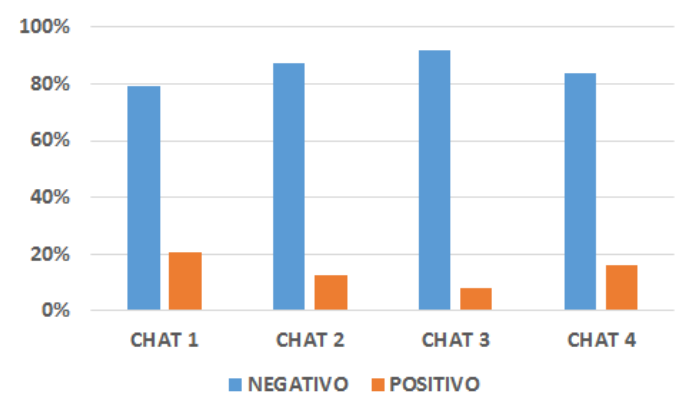

(b) Percentual de acertos sobre o total de mensagens identificadas corretamente

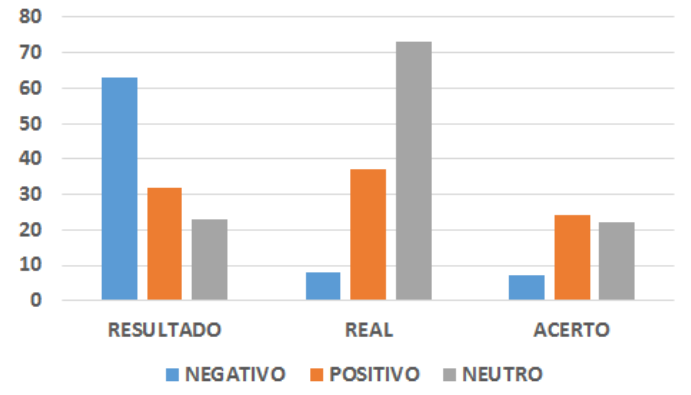

(d) Quantidade de mensagens para o chat 1 recalculado com o nível neutro

Figura 1. Sumário das mensagens para todos os chats

A Figura 2 exibe gráficos apenas para o experimento com o chat 1 recalculado. A Figura 2(a) apresenta a quantidade de acertos obtidos para os três níveis, a Figura 2(b) exibe o percentual de acertos sobre o total de mensagens identificadas corretamente, e a Figura 2(c) exibe o percentual de acertos sobre o total de mensagens. 
V Congresso Brasileiro de Informática na Educação (CBIE 2016)

Anais dos Workshops do V Congresso Brasileiro de Informática na Educação (CBIE 2016)

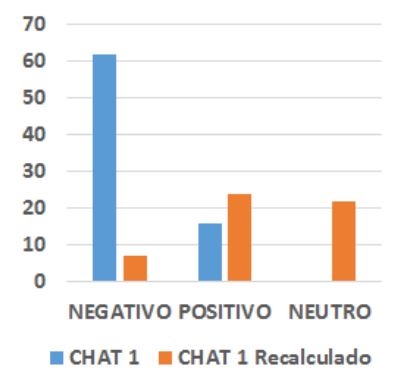

(a) Quantidade de acertos obtidos

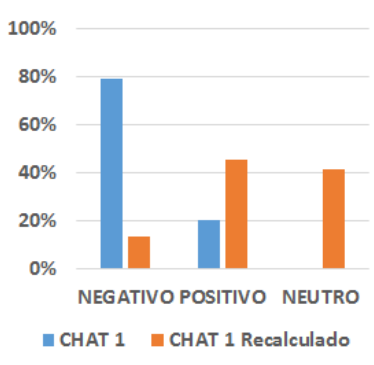

(b) Percentual de acertos sobre o total de mensagens identificadas corretamente

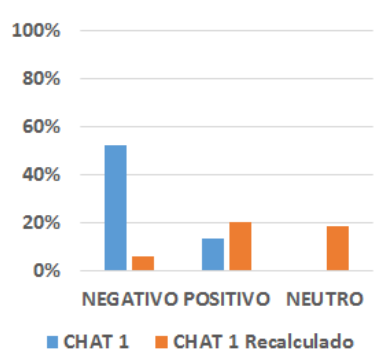

(c) Percentual de acertos sobre o total de mensagens

\section{Figura 2. Sumário das mensagens para o experimento com o chat 1 recalculado}

\subsection{Análise do Resultados}

De maneira geral, e analisando a taxa de acerto do sentimento das mensagens, e considerando o pequeno tamanho da base de treinamento (apenas 113 palavras), considera-se que esse experimento obteve sucesso.

Percebe-se pelas figuras que a maioria dos acertos foi do tipo negativo. Entretanto para os experimentos que possuíam apenas dois níveis (positivo e negativo), a maioria das palavras foram taxadas como negativas por não conseguirem ser classificadas, e muitas delas não possuíam essa polaridade. No experimento com três níveis esse fato não ocorreu, pois a maioria das palavras foi classificada manualmente como neutras, e isso impactou nos resultados do algoritmos, deixando a classificação mais real. Percebe-se aqui a importância de um dicionário com classificações precisas.

Um problema que se identificou foi a presença de "emoticons" ao longo das mensagens. Essa presença é comum em chats, e praticamente todas suas ocorrências ou foram classificadas como negativas ou neutras, pois não havia nenhuma representação para elas na base de treinamento. A presença de gírias e abreviações de palavras também prejudicou a identificação do sentimento, pois o algoritmo não conseguiu classificá-las corretamente. Muitos termos da internet, como "blz" e "vlw" foram também utilizados nas mensangens, não sendo também classsificados corretamente.

Um dos motivos para se utilizar análise de polaridade positiva e negativa em chats foi estudar a técnica, para poder avaliar sua utilidade e aplicabilidade em outros aspectos de um AVA. Concluiu-se como sendo uma técnica bastante útil. Não foi investigado se o aluno compreendeu ou não um determinado assunto ou conceito, pois o foco realmente foi avaliar a técnica e como as discussões estavam fluindo nos chats.

Também não foi executado nenhum pré-processamento no texto, necessitando de um estudo maior na técnica. Logo, não considerou-se a análise de stopwords. Stopwords são palavras muito comuns que aparecem no texto e não possuem muito significado, servindo apenas para funções sintáticas, não indicando importância ao assunto [El-Khair 2006]. Exemplos de stopwords são: as, os, de, e, para e com.

Em um projeto real mais amplo, onde há uma grande quantidade de mensagens, interações, diferentes perspectivas, o conjunto de dados de treinamento deve conter muito mais dados. Entretanto, para o objetivo do experimento, que foi explorar a ideia de anali- 
V Congresso Brasileiro de Informática na Educação (CBIE 2016)

Anais dos Workshops do V Congresso Brasileiro de Informática na Educação (CBIE 2016)

sar o sentimento de mensagens de chat, acredita-se que este foi atendido.

Em relação ao teor dos chats, analisando os textos, o que se percebeu é que a maioria das mensagens possui características neutras. Alguns comportamentos que foram capturados pelo algoritmo foram:

- Muitos dos acertos corresponderam a sequências de "boa noite", e como este conjunto de palavras é fácil de se identificar, muitos acertos ocorreram por este fato, que no caso é uma mensagem positiva;

- O início das sessões do chat geralmente possuiu várias mensagens de apresentação e entrada no chat, com mensagens cordiais, também classificadas como mensagens positivas;

- O término das sessões do chat geralmente possuiu várias mensagens de despedida, com mensagens de motivação, em geral classificadas como mensagens positivas.

\section{Conclusões e Trabalhos Futuros}

Este trabalho apresentou um experimento de análise do sentimento em mensagens de chats. O objetivo foi realizar uma avaliação preliminar com um algoritmo conehcido de aprendizado de máquina (Naive Bayes), e aplicá-lo na identificação do sentimento de alunos por meio de mensagens de chat.

Um estudo de caso foi realizado em quatro chats de um curso semi-presencial, com mensagens capturadas de um AVA. As principais conclusões foram que é aprendizagem de máquina é altamente aplicável no contexto educacional, que diversos fatores influenciam na identificação do sentimento de texto, tais como abreviações de palavras, emoticons e gírias. Além disso, a necessidade de uma base de dados para treinamento com qualidade também é essencial.

Ainda se torna necessário utilizar a técnica de análise de esntimento em tempo real, pois assim se obteria o real benefício de uma avaliação de chats e fóruns e as intervenções poderiam ser mais efetivas. Como trabalhos futuros, pretende-se: (i) realizar mais experimentos para a identificação de emoticons, gírias e abreviações de palavras; (ii) criação de um dicionário (base de dados) de sentimentos para termos da internet utilizados com frequência em chats; e (iii) avaliar diferentes estratégias de classificação.

\section{Referências}

Araújo, M., Gonçalves, P., and Benevenuto, F. (2013). Métodos para análise de sentimentos no twitter. In 19th Brazilian Symposium on Multimedia and the Web (WebMedia2013).

David, P. B., de Lima, E. T., and Mendes, F. A. G. (2015). Uma ontologia de domínio para a análise do diálogo freireano em fóruns de discussão da educação a distância. In CBIE-LACLO 2015 - Anais do XXVI Simpósio Brasileiro de Informática na Educação (SBIE 2015).

David, P. B., Freire, R. S., de Lima, E. T., Paillard, G. A. L., Viana, W., and Mendes, F. A. G. (2014). Análise da afetividade em fóruns virtuais: Construção de uma ontologia de domínio. In Nuevas Ideas en Informática Educativa (TISE 2014), pages 821-826. 
V Congresso Brasileiro de Informática na Educação (CBIE 2016)

Anais dos Workshops do V Congresso Brasileiro de Informática na Educação (CBIE 2016)

Dosciatti, M. M., Ferreira, L. P. C., and Paraiso, E. C. (2013). Identificando emoções em textos em português do brasil usando máquina de vetores de suporte em solução multiclasse. In ENIAC - Encontro Nacional de Inteligência Artificial e Computacional.

El-Khair, I. A. (2006). Effects of stop words elimination for arabic information retrieval: a comparative study. International Journal of Computing and Information Sciences, 4(3):119-133.

Gomes, H., de Castro Neto, M., and Henriques, R. (2013). Text mining: Sentiment analysis on news classification. In 2013 8th Iberian Conference on Information Systems and Technologies (CISTI), pages 1-6.

Li, G. and Liu, F. (2010). A clustering-based approach on sentiment analysis. In Intelligent Systems and Knowledge Engineering (ISKE), 2010 International Conference on, pages 331-337.

Liu, B. (2012). Sentiment analysis and opinion mining. Synthesis Lectures on Human Language Technologies, 5(1):1-167.

Malheiros, Y. and Lima, G. (2013). Uma ferramenta para análise de sentimentos em redes sociais utilizando o senticnet. In IX Simpósio Brasileiro de Sistemas de Informação (SBSI2013).

Missen, M. M. S., Boughanem, M., and Cabanac, G. (2013). Opinion mining: reviewed from word to document level. Social Network Analysis and Mining, 3(1):107-125.

Oliveira, V. M. and Roman, N. T. (2013). Jwn-br - uma api java para a wordnet.br. In 9th Brazilian Symposium in Information and Human Language Technology, pages 153157.

Owen, S., Anil, R., Dunning, T., and Friedman, E. (2011). Mahout in Action. Manning Publications Co., Greenwich, CT, USA.

Reis, J. C. S., Gonçalves, P., Araújo, M., Pereira, A. C. M., and Benevenuto, F. (2015). Uma abordagem multilíngue para análise de sentimentos. In IV Brazilian Workshop on Social Network Analysis and Mining (BraSNAM 2015).

Rennie, J. D. M., Shih, L., Teevan, J., and Karger, D. R. (2003). Tackling the poor assumptions of naive bayes text classifiers. In In Proceedings of the Twentieth International Conference on Machine Learning, pages 616-623. 